Franklin, BD; (2014) Medication errors: do they occur in isolation? BMJ Quality \& Safety , 23 (5), Article e1. 10.1136/bmiqs-2014-002924.

\title{
EDITORIAL
}

\section{Medication errors: do they occur in isolation?}

\author{
B D Franklin
}

Centre for Medication Safety and Service Quality, Imperial College Healthcare NHS

Trust/UCL School of Pharmacy, London WC1N 1AX, UK; bryony.deanfranklin@imperial.nhs.uk

Medication error research typically focuses on just one stage of the medication use process: prescribing, transcribing, dispensing, administration or monitoring. Such simplification is required so that manageable boundaries can be set around the research, and to make it easier to specify error definitions, methods and denominators. However, this is clearly very simplistic, and from the patient's perspective it is a somewhat arbitrary and meaningless distinction. In addition, many quantitative studies present the numbers of doses, drugs or patients with one or more errors, which makes it easy to miss the fact that multiple errors can occur together. While studies of adverse drug events (ADEs) typically include a wider range of sources of harm, some analyses include only the first ADE identified for each patient. Collectively, this means we may miss the opportunity to explore how multiple errors may occur together in the same patient. And yet detailed investigations into catastrophic medication errors typically reveal a whole concatenation of errors and failed defences, involving multiple stages of the medication use process. $(1,2)$

We therefore need to better understand how errors are related across the whole medication process. A paper presented by Carayon et al(3) explores this very issue in two US adult critical care units, shedding new light on the complexities surrounding medication use in the hospital setting. While the critical care setting is likely to be particularly complex, with patients prescribed high numbers of medications, particularly intravenously, the principles are likely to apply much more widely.

Carayon et al took the novel approach of analysing errors both by group (where more than one error occurs for the same medication order at the same stage of the medication use process) and sequence (where errors in one stage of the medication use process were judged to have led to further errors in other stages). They identified medication errors and ADEs in 630 critical care admissions, with their main analysis focusing on the more serious errors: potential ADEs (medication errors which could have resulted in harm but did not) and preventable ADEs (medication errors which resulted in actual patient harm). There were 1145 potential ADEs and 38 preventable ADEs. Overall, most (84\%) of these involved single medication errors, but $6 \%$ involved grouped errors and $10 \%$ sequential errors.

Of the 145 grouped errors involved in 67 potential or preventable ADEs, almost all occurred at the ordering (prescribing) stage, where information on several elements of the same medication order was missing or incorrect. These are not discussed further in the paper, but such grouping might suggest the prescription of medication for which the prescriber had limited knowledge, was rushing, or was otherwise distracted.

In contrast, the most common sequences involved an error in dispensing followed by an error in administration (usually omission of medication), or an error in transcription followed by an error in administration due to medication administration records being incorrect. 
Many other sequence combinations were also observed: the 121 potential and preventable ADEs caused by sequential errors involved a total of 264 errors and 21 different sequences. The majority of sequences culminated in an error at the administration stage but most began at ordering, transcription or dispensing. Staff responsible for administering medication are therefore 'inheriting' a wide range of errors from earlier stages of the medication use process.

While no previous studies provide such a detailed description of grouped and sequential medication errors across the whole medication use process, reports from other countries also highlight how errors in one part of the process can lead to others downstream. Taxis et al(4) identified medication administration errors using observation in medical and surgical wards in two German hospitals and a UK hospital. They classified errors according to the stage of the system in which they were considered to originate, demonstrating key differences between the three hospitals studied. In the UK hospital, which used a typical British ward pharmacy system, errors mainly originated at the administration stage, with others due to delays in drugs being ordered and supplied from the pharmacy. In the two German hospitals, one using unit dose dispensing and one a more traditional European medication system, most errors originated in transcribing. In all three contexts, while very different to the setting described by Carayon et al, nurses administering medication were inheriting errors from other parts of the system. In another very different setting, Silva et al(5) studied dispensing and prescribing errors in high risk medicines used for paediatric inpatients in a Brazilian hospital, and analysed the extent to which dispensing and prescribing errors occurred together in the same prescriptions. Of 1707 dispensing errors identified, $42 \%$ were considered to have arisen due to prescribing errors, usually concerning medication being dispensed without the dosage form and/or dose being specified by the prescriber.

So what are the implications of such findings? First, medication errors in general and administration errors in particular can often be caused by breakdowns in earlier stages of the medication use process, a point which should be taken into account when identifying potential solutions. Furthermore, while pharmacists and nurses are able to play an important role in identifying and rectifying prescribing and transcribing errors, reducing the numbers of such errors in the first place is likely to be a more effective and efficient approach.

Second, we need to understand how changes in the medication use process, such as the introduction of various information technologies, affect the types and causes of medication errors as well as their prevalence. Carayon et als study was conducted prior to the introduction of electronic health records and computerised prescriber order entry (CPOE). While there is a considerable literature about the effects of CPOE on the prevalence of medication errors and $\operatorname{ADEs}(6)$, its effects on communication and complexity are less well documented. A comparable analysis following the introduction of electronic health records and CPOE will be an important addition to the literature. There is also scope for further types of sequential errors with CPOE-one error made in setting up a default dose or order set can potentially affect thousands of patients.

Third, the study provides further evidence of the importance of using a range of methods to identify medication errors. Only $2 \%$ of all events were identified via the hospital's incident reporting system; reviewing charts and missing dose reports revealed the majority. Relying on incident reports to elicit such data would have therefore provided a very incomplete picture. However, even in this comprehensive study, it is possible that other errors were missed. Data were collected by trained nurses who reviewed all relevant 
documentation as well as inviting reports of adverse events from clinicians. However, this approach is unlikely to have identified medication administration errors or dispensing errors other than those resulting in omission or delay; observation of medication dispensing or administration may have revealed further errors. It is also possible that a specialist critical care pharmacist or physician would have identified different errors. The true picture may be even more complex than described.

Finally, Carayon's study focused only on two clinical units. Even within these units, considerable complexity has been revealed. And yet an individual patient's healthcare journey is likely to involve medication being prescribed, dispensed and administered across the whole range of healthcare contexts, including their own home as well as various hospital settings. There is therefore considerable scope for much more complex error sequences to occur than those described here.

In conclusion, it is clear that medication errors do not always occur in isolation. While detailed studies of individual stages of the medication use process are useful in fully characterising specific types of errors, we also need to understand how errors inter-relate across the entire healthcare system, how and why they occur, and how they can be prevented. From the patient's perspective, this is likely to be what matters most.

\section{Footnotes}

- Funding The Centre for Medication Safety and Service Quality is affiliated with the Centre for Patient Safety and Service Quality at Imperial College Healthcare NHS Trust which is funded by the UK National Institute of Health Research (NIHR) as a Patient Safety Translational Research Centre.

- Competing interests None.

- Provenance and peer review Not commissioned; internally peer reviewed.

- This editorial is published online only and comments on a paper published in the January print issue of the journal.(3)

\section{References}

1. Toft B, . External inquiry into the adverse incident that occurred at Queen's Medical Centre, Nottingham, 4th January 2001. http://www.who.int/patientsafety/news/Queens\%20Medical\%20Centre\%20report\%2 O(Toft).pdf (accessed 19 February 2014).

2. Cambridgeshire Health Authority. Methotrexate toxicity: an inquiry into the death of a Cambridgeshire patient in April 2000. 2000.

http://www.blacktriangle.org/methotrexate-toxicity.pdf (accessed 19 February 2014).

3. Carayon P, Wetterneck T, Cartmill R, et al. Characterizing the complexity of medication safety using a human factors approach: an observational study in two intensive care units. BMJ Qual Saf 2014;23:56-65.

4. Taxis K, Dean BS, Barber ND, Hospital drug distribution systems in the UK and Germany - a study of medication errors. Pharm World Sci 1999;21:25-31.

5. Silva MDG, Rosa MB, Franklin BD, et al, Concomitant prescribing and dispensing errors at a Brazilian hospital: a descriptive study. Clinics 2011;66:1691

6. Ammenwerth E, Schnell-Inderst P, Machan C, et al, The effect of electronic prescribing on medication errors and adverse drug events: a systematic review. $J$ Am Med Inform Assoc 2008;15:585-600. 